\title{
Opinion paper: 'monitoring te salutant:' combining digital sciences and agro-ecology to design multi-performant livestock farming systems
}

\author{
S. Ingrand ${ }^{\dagger}$ \\ Université Clermont Auvergne, AgroParisTech, Inra, Irstea, VetAgro Sup, UMR Territoires, F-63000 Clermont-Ferrand, France
}

First published online 22 August 2017

Intensive livestock farming is receiving considerable criticism, especially in Europe where the current systems are associated with low animal welfare and negative environmental impacts. For several years, the research agenda has been targeting ways to increase the sustainability of livestock systems, that is, to improve their societal and environmental performances without decreasing the economic outcome, in other words to design multi-performant systems. The central message of this text is that the efficient way to achieve this goal is to combine both agro-ecology and digital sciences.

Agro-ecology consists of bringing together ecology and agronomy, that is, enhancing ecological processes and regulations to increase both yields and robustness (Dumont and Bernues, 2014, Special issue of Animal). Five goals need to be considered in order to move toward more agro-ecological livestock farming systems: the integrated management of animal health, low level of pollution (air, water, soil), low level of inputs, high adaptive capacity, thanks to diversity within the system and a high level of biodiversity Dumont et al. 2013.

Digital sciences offer new possibilities to monitor and to drive livestock systems. The EU-PLF (precision livestock farming; www.eu-plf.eu) project and the very recent creation of a specific new study commission at the European Federation of Animal Production (www.eaap.org) dedicated to PLF systems are just examples for the fast development of this exciting new field. Farmers are increasingly relying on new digital technologies and communication. Recording of environmental, biometric and physiological parameters as well as behavioral characteristics of animals provide data which are all elements of decision support for precision farming, that is, adjustment of technical management practices to be as close to the individual animals' needs as possible in order to achieve the farmers' objectives (Halachmi and Guarino, 2016).

Combining agro-ecology and digital sciences would minimize the risks of failure for both of them than if they are

\footnotetext{
${ }^{\dagger}$ E-mail: stephane.ingrand@inra.fr
}

developed separately. For agro-ecology, the risk is a lack of capacity to motivate different actors: researchers, farmers and all stakeholders, because it could appear for them as a step backwards, a kind of old-fashioned way of managing systems. On the other hand, this is quite acceptable for consumers and from a societal point of view. For digital sciences and new technologies, the risk is to make researchers enthusiastic but to be rejected by society, because of the perception of some kind of mechanical system, associated with a lot of captors, without seeing the farmer anymore in the barn.

To succeed in combining digital sciences and agro-ecology, there is a prerequisite for researchers, which is to clarify the difference between PLF on one hand and high-throughput phenotyping on the other. They are both based on captors and automated procedures, but the objectives are not the same (Figure 1). High-throughput phenotyping concerns precise, repeated and frequent measurement of traits. This is more and more feasible, thanks to the development of accurate technologies (miniaturized captors, software empowerment, etc.), with the principal objective to produce knowledge in biological sciences, using a 'data-driven' process. The objective of PLF is to improve the management and the monitoring process at the animal scale. This implies associating farmers' goals and strategies, to a set of rules concerning management. In other words, the role of captors is to provide information, whereas the role of farmers is to make decisions.

Knowledge about biological science is crucial in this process and should be used as a driver (i) to better understand what traits and data to monitor, (ii) to determine what rules are appropriate to make decisions using those data. Hence, captors should never make unsupervised decisions and will never replace the farmer's skills. They 'only' provide data to nurture the decisions and can eventually be programmed to apply automated procedures, according to farmer's strategies; that is, the important part of decision support tools is not the 'decision' but the 'support'-ing information. The large amount of data provided are also useful for researchers in a 


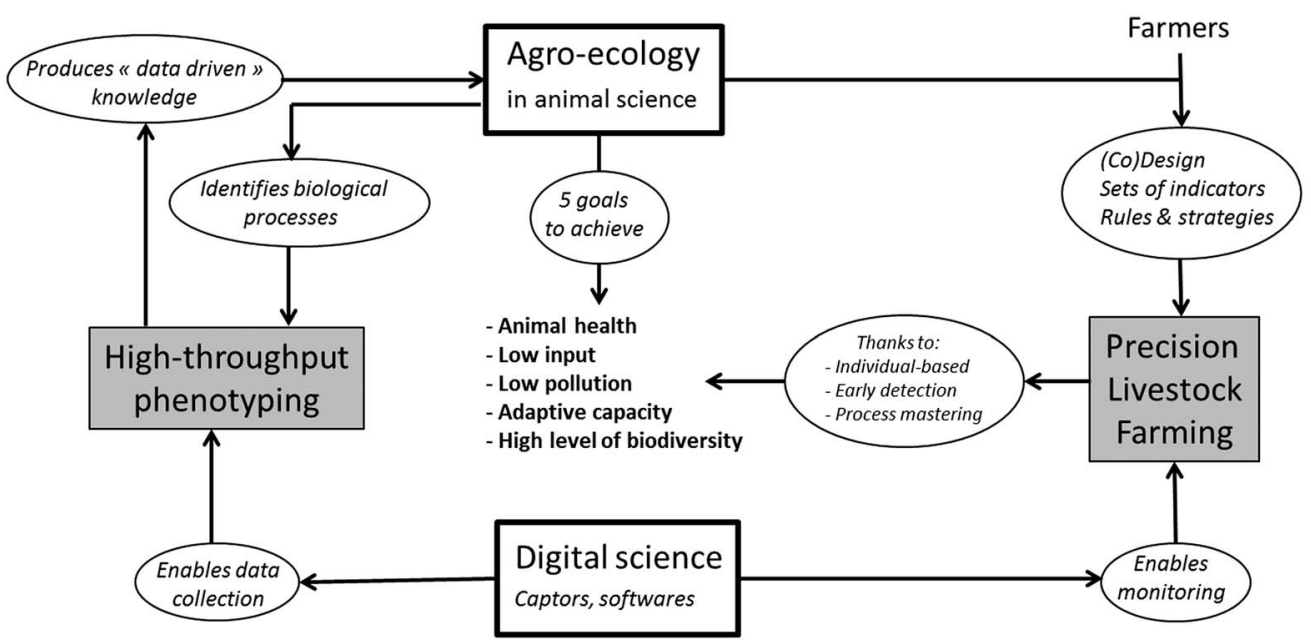

Figure 1 Bringing together digital sciences and agro-ecology to design multi-performant livestock farming systems, thanks to livestock precision farming.

'data-driven process,' allowing them to make the link between processes, that is to define biomarkers able to predict biological parameters. This is the case, for example, with internal body temperature, when monitored continuously by using a transponder placed within the animal's body, which allows the detection of very early signs of a dysfunction (illness, including mastitis in dairy herds).

Overall, this means that biology and technology will be the two essential levers that have to be activated jointly. The exciting challenge is then to bring together agro-ecology and technology (digital sciences) to enhance the sustainability of livestock farming systems in the future, that is to invent agroecology 3.0, which is often considered as an oxymoron!

This concerns both intensive (e.g. poultry, pigs, fishes) and extensive systems (ruminants in grassland areas).

An example for intensive systems combined with agroecological goals is an on-going project at Inra, which consists of designing 'hi-tech' organic pig experimental facilities. The main idea is to propose facilities to researchers, with freerange sows and fattening pigs, representing an alternative to the current conventional system, but with captors and all the technology to gather individual-based data from the system. This will allow us to show that new digital technologies enable the development of extensive systems which are as good as conventional housing systems for technical performances while being better for animal welfare.

In extensive grass-based systems, the lack of individual monitoring is often a bottleneck to increase the efficiency of the system. An example is another project on free-ranging sheep in pastoral areas from the south of France. The aim is to understand and to predict the herd's behavior for a better grazing management and to detect unexpected behavior. This is done by using 'sentinel' animals equipped with transponders measuring the distance with the other equipped counterparts.

To conclude, combining agro-ecology and digital sciences should effectively contribute to achieve the five goals in order to move toward more agro-ecological livestock farming systems. Digital technologies allow old-fashioned oversight of every animal and, more widely, of every component of the whole system, to be brought back in, in the modern age. This new capacity for monitoring every component of the system is a huge lever to increase the overall efficiency, and to better manage health, to avoid pollution, to decrease inputs and to manage diversity as an added-value rather than a constraint. This contributes to a higher adaptive capacity of the system as well as a high level of biodiversity.

Combining agro-ecology and digital sciences is a great opportunity to give accurate responses to current and future societal demands and last, but not least, to make animal husbandry more attractive to young farmers.

\section{References}

Dumont B and Bernues A (eds) 2014. Special issue: Agroecology: integrating animals in agroecosystems. Animal 8, 1201-1393.

Dumont B, Fortun-Lamothe L, Jouven M, Thomas M and Tichit M 2013. Prospects from agroecology and industrial ecology for animal production in the 21st century. Animal 7, 1028-1043.

Halachmi I and Guarino M 2016. Precision livestock farming: a 'per animal' approach using advanced monitoring technologies. Animal 10, 1482-1483. 